\title{
La formation des élites en tant que problématique d'ordre socioéconomique dans le développement local
}

\author{
Driss AAYADI
Etudient chercheur à l'EMI: Impact de
la culture sur la qualité
}

Mots clés: management, formation, élites, sociologie

\section{Résumé}

Le secteur automobile est un des secteurs industriels décidés par le plan émergence Marocain très prometteur pour les investisseurs, aussi il offre une possibilité de développement très intéressante vis-àvis des stratégies de croissance des entreprises visant l'internationale.

Renault et d'autres équipementiers automobiles ont choisi de s'installer au Maroc pour exercer leurs métiers et activités de production, cependant ces firmes auront besoin des élites locales qualifiées pour accompagner leurs développements sur le plan national.

Pour répondre à cette problématique à dimension socioéconomique. Le présent essai vient pour mettre en évidence, les dimensions culturelles Marocaines qui peuvent influencer la formation des futures élites Marocaines et cadre locales en la matière de l'automobile.

Il est à noter que toute entreprise délocalisée visant le développement et l'innovation de ces activités dans les pays d'accueil devra prendre en compte ces dimensions dans sa stratégie et ces plans de formation dédiés à la bonne gouvernance de la montée en compétence de ces élites. 


\section{Introduction}

$\grave{A}$ l'aube de cette mondialisation et externalisation, les entreprises deviennent de plus en plus combatives et compétitives. Elles visent en conséquence, s'approprier le meilleur niveau de la qualité possible de leurs produits en se comparant à d'autres concurrents exerçant le même métier ou opérants dans le même secteur d'activité. Pour cela, on utilise les différentes méthodes les plus efficaces et les plus expérimentées en la matière des pratiques organisationnelles ainsi qu'en management de la qualité, dans un but global de faire bon du premier coup et par la suite satisfaire les besoins du client dans les plus brefs délais et avec la meilleure qualité requise, tout en veillant à un prix concurrentiel et compétitif.

En revanche, cette mobilisation reste insuffisante pour atteindre l'excellence industrielle et les marges de gain souhaitées. Ceci dit les entreprises notamment les multinationales se retrouvent dans l'obligation de prospecter d'autres opportunités et chercher d'autres pistes pour améliorer leurs performances.

Renault comme d'autres constructeurs ou équipementiers automobiles a choisi la piste d'externalisation des activités vers les pays émergents comme le Maroc, dans un objectif stratégique qui est la réduction des coûts de production. En conséquence la firme prend en charge la formation de ces futures élites locales qui assureront par la suite le développement local et l'innovation des activités que ça soit : 
l'innovation en produit, l'innovation en procédés de fabrication, l'innovation en organisation ou aussi l'innovation en marketing.

\section{Contextualisation du sujet}

Pour débuter cet essai, il s'est avéré essentiel de rappeler une définition de la culture parmi les 164 définitions qui ont été recensés par Kloeber et Kluckholm. Pour Barnouw (1963) la culture est définie comme étant :

« le mode de vie d'un groupe d'individus, la combinaison de tous les modèles plus ou moins stéréotypés de comportement appris, que chaque génération transmet à la suivante au moyen du langage et de l'imitation ».

Cette définition regroupe plusieurs autres définitions qui renvoient le concept de la culture à la notion du système de communication et annonce par la suite une multitude d'approche dans ce sens. Pour Hall(1984) la culture est un « système de communication et de traitement de l'information», aussi Hofstede (1980) traite «la culture comme une sorte de programmation mentale gérée par l'apprentissage continu ».

À partir De cette première introduction, on voit que l'analyse du comportement sociologique au MAROC et plus spécifiquement les élites Marocaines devra passer par une discrétisation locale et régionale du pays, pour amener ensuite une démarche de liaisons avec le contexte émergent du tissu industriel Marocain, ainsi que de savoir les interactions, les échanges et les exigences des secteurs en 
développement régional par rapport au comportement de la population locale.

\section{Eléments culturels Marocains influençant la formation des élites locales}

Le contexte Marocain est un contexte tellement riche qu'on parlera d'échange de l'information à plusieurs niveaux sociétaux qui ont quelques points de repère fixes en temps et en espace constituant des points de convergences repérables pour cerner la vie sociale au Maroc.

Sur le plan constructif culturel de notre pays, le Maroc est un pays d'échange culturel par excellence et de diversité socioculturelle très important et assez intense. Ceci, est dû à de nombreux facteurs sur plusieurs horizons et plans. D’un point de vue géographique, Le Maroc a une situation géographique phare au niveau de l'échange et du déplacement des personnes entre les Quatre coins du monde, et spécifiquement une traversée privilégiée de l'Afrique du côté Sud de la terre vers l'Europe et les pays du Nord, ainsi qu'une porte commerciale de l'Est vers l'Atlantique et l'Ouest dans sa globalité.

En addition à cela, Le Maroc fût un point de rencontre des différentes routes commerciales vers l'Est. On ajoute encore le nombre important des civilisations qui ont envahi le Maroc et qui ont laissé des traces culturelles lors de leurs passages dans le pays. Depuis l'arrivée des Arabes en 682, « arrivé d'Oqba Ibn Nafi'i » et le commencement 
de l'islamisation du pays, jusqu'à l'arrivée de la colonisation Française au centre du Maroc et celle Espagnole au sud et au Nord Est du pays.

Sur ce, la culture Marocaine est constituée d'ensemble de précipitées culturelles qui ont formé un mélange culturel important. Donnant une mosaïque culturelle au pays, constitué de plusieurs éléments culturels constitutifs, constructifs et inséparable.

\section{Dimensions culturelles universelles et dimension culturelles spécifique au contexte Marocain.}

Les dimensions culturelles universelles les plus connues et qui sont fondamentales pour catégoriser une culture par rapport à d'autres cultures, sont regroupées en trois grandes catégories :

Pour Hofstede (1980), on pourra caractériser une culture nationale selon quatre grandes valeurs, appelées aussi dimensions culturelles : la distance hiérarchique, le contrôle de l'incertitude, le degré d'individualisme ou de collectivisme et le degré de masculinité ou de féminité dans une société. Plus tard, Hofstede (1991) ajoutera une cinquième dimension : l'orientation vers le court ou le long terme d'une société.

Selon Hall(1984), on propose trois dimensions "cachées" dans un système : le contexte (Contexte riche versus contexte pauvre), le temps (Temps monochrone versus temps polychrone) et l'espace (Proxémique culturelle). 
D'autres auteurs ont proposé plusieurs autres visions de caractérisation culturelles ainsi que d'autres dimensions culturelles. Pour Trompenaars et Hampdon-Turner (2004), la culture est considérée comme un ensemble d'universaux partant du principe que chaque culture propose ses propres solutions pour résoudre les problèmes et dégage par la suite sept dimensions culturelles. D'Iribarne (2002) propose une base d'idée selon ces enquêtes, à partir d'un jumelage de l'ethnographie avec les systèmes d'organisation sociale et politique.

Nous allons essayer dans ce qui suit, de contextualiser ces dimensions culturelles au contexte Marocain, pour aboutir à de nouvelles propositions de dimensions culture qui apparaît mieux adaptée à ce contexte et à sa composition de sous-culture fondés sur une composition ethnographique par excellence.

\section{Influence des dimensions culturelles dans la formation des élites locales}

La stratégie de la formation des élites dans le monde de l'automobile dépende largement de l'entreprise elle-même et à ces plans de formation prévus pour ces cadres. Au Maroc l'activité de l'automobile étant nouvellement installée, les entreprises délocalisées se trouvent dans l'obligation de constituer leurs propres centres de formation et d'accompagnement de leurs futures cadres en automobile.

Sur ce Renault a choisi d'investir en sa capitale humaine locale et de construire en partenariat avec l'État Marocain l'Institut de formation aux métiers de l'Industrie Automobiles (IFMIA), dédié à la formation 
des élites de l'automobile Marocaine. Cette action a constitué un grand pas vers l'industrialisation du pays et a sa mise en route parmi les leadeurs de l'activité automobile à l'échelle mondiale.

On note aussi que ce succès dépend de la vise stratégique de la direction qui a pris en compte les valeurs Marocaines dans toute sorte de formation prévu pour les cadres locales.

En fait pour la même raison Soussi(2008), a critiqué les propos de D'Iribarne(2002) sur l'interaction positive du Marocain musulman avec la démarche qualité de la TQM (Total Quality Management).

Pour D'Iribarne (2002) l'application de la TQM (Total Quality Management) au sein de l'entreprise de Sgs Thomson, représente un mode de management ainsi qu'un système d'organisation sociale d'une part, et l'Islam d'autre part, comme une religion qui règne pour une ethnographie et une race humaine bien spécifique.

L'expérience a eu un grand succès dû au plan stratégique de la formation sur la TQM qui prend en compte la nature de la culture hautement coopérative des Marocains musulmans et une stratégie d'insertion bien étudier.

Pour Soussi(2008), le succès a bien eu lieu étant un résultat direct de l'échange qui a eu lieu entre le manuel de la qualité de la TQM et les dimensions culturelles qui gèrent les sous-cultures Marocaines pour former les élites dans le domaine d'activité de la société.

Allali (2008) affirme qu'il y a mainte valeur humaine qui gère la culture Marocaine et qui constituent les dimensions du culturalisme 
Marocain, ainsi ces derniers ont une influence directe sur les modes de gestion industrielle (Management Industriel) adopté au MAROC.

Pour Soussi(2008), Trois valeurs ou (Dimensions culturelles) justifient bien le comportement des Marocains vis-à-vis de cette application de la TQM au sein l'entreprise Sgs Thomson et non pas les propos de (D'Iribarne, 2002) et (Geertz, 1979) sur l'interaction de l'Islam comme référence morale et les principes de la (TQM) comme mode de management, testé, appliqué avec succès au Maroc.

Allali (2008) a spécifie les trois dimensions suivantes pour étudier la culture Marocain :

1. La logique d'allégeance, qui présente une interaction entre la religion et le mode de management ainsi qu'une vision d'individualisme dans une société catégorisée par Hofstede (1980) de société collectiviste, ce qui contredit les propos de D'Iribarne (2002) dans son analyse chez Sgs Thomson.

2. la dimension familiste qui se trouve au carrefour de l'individualisme et le collectivisme, cette dernière étant une dimension, bien citée et prise en considération dans les modes de management des entreprises Marocaines. Ces entreprises généralement pilotées par deux grands groupes Marocains, ceux des Fassi d'une part et des Soussi de l'autre part.

Mamou(1985), parle même d'un mode de gestion issu du familialisme, celui de l'entrepreneur Paternaliste, elle enchaine «... Par contre, la solidarité, dans la société soussie est à la base de toutes les 
raisons sociales. Le Soussi a besoin de faire partie d'un groupe... ». Ainsi (Saadi, 1982), en parlant des modes de gestion de l'entreprise Marocain, Panéliste/Familialisme, définit ces modes comme : «... la monopolisation de l'encadrement supérieur par les membres de la famille pour des considérations qui n'ont rien à voir avec la compétence... » .

3-En 3ème lieu, Allali(2008) spécifie la sacralité de la dignité comme les 3èmes dimensions culturelles Marocaine, qui constitue une dimension vitale pour les Marocains, vu leurs positions historiques contre les colonisations et protectorat ainsi le nombre d'entreprises qui ont rendu les clés, étant une conséquence directe de leurs modes de management autoritaire qui contredit cette valeur enracinée dans la culture Marocaine, et qui ne prend pas en compte : la dimension du contrôle de l'incertitude, affirme Saadi(1982) sur les failles du système Paternaliste, «...la routine empirique peu créative, l'insuffisance des moyens de contrôle à court terme, l'absence d'orientation à long terme».

En conclusion la prise en compte des dimensions culturelles locales a une vocation essentialiste dans la formation des élites des métiers, leurs insertions, leurs accompagnements et leurs montées en compétence.

Comparaison a d'autres cultures émergentes et questions de consolidation des résultats

$\mathrm{Au}$ Maroc, on se dote de plusieurs valeurs qui gèrent nos aspects culturels, et qui rentre dans le système d'interaction avec le type de 
management, d'organisation adaptée et ainsi le mode de formation choisi pour les élites.

L'expérience de la réussir de l'application du mode de management de la qualité nommé : $\mathbf{T Q M}$ adopté par Sgs Thomson et la formation du personnel qualifiés dans ce mode de gestion, a donné une vision essentialiste de l'islam ainsi que ses valeurs comme la logique d'allégeance, la sacralité de la dignité et la dimension familiste dans la texture culturelle Marocaine. Sur la même voie, l'application du mode de management nommé : «double projet », dans la filiale Mexicaine de Danone a donné les mêmes conséquences positive en terme de valeurs humaines, puisque le but du projet et de bâtir une politique humain saine en parallèle à la productivité, la profitabilité et la rentabilité de l'entreprise. On pourra parler donc d'une vision familialiste de l'environnement de l'entreprise dans son ensemble, reflétée par l'utilisation d'un seul langage le «nous », et le faite de représenter une seule image de l'entreprise et ces équipes vis-à-vis de l'environnement externe à l'entreprise, ce qui n'existe pas forcément dans d'autre filiale. En plus de ces deux expériences, s'ajoute celle du Vietnam qui a montré deux caractéristiques contradictoires de la culture vietnamienne concernant, les dimensions du collectivisme et du Power Distance. Ainsi on a montré la nécessité de la contextualisation du mode de management de l'entreprise au contexte culturelle local pour réussir l'expérience du management issu du siège sociale et la position essentiel de la formation des référents locaux. 


\section{REFERENCE}

Allali B., (2008) «Culture et gestion au Maroc : une osmose atypique ». Québec, Presse de l’Université Laval et Télé-Université (UQAM).

Labari B., (2011) «Islam et Entreprise : Référent Divergeant et Rationalités Contestataires ». Revue : Mandrágora, v.17. n. 17, 2011, p. 83-97.

D'Iribame, Ph. (2007) «Islam et management. Le rôle d'un univers de sens»,| Lavoisier | Revue française de gestion, 2007/2 - n 171, p. 141156.

D'Iribame, Ph. (2002) «Motivating workers in emerging countries: universal tools and local adaptations», Joumal of Organizational Behavior. 23, p. 243-256.

Sadik, S. (2002) «L'approche culturelle et le Management par la Qualité Totale «TQM » dans le contexte marocain » Revue : Repères et Perspectives - $\mathrm{N}^{\circ} 3$, Automne 2002.

D’Iribame, Ph. (2001) «Un management modérne enraciné dans une culture traditionnelle, Les enseignements d'une success-story mexicaine »,Revue : Gérer et Comprendre, 09/2001 , N 65.

D'Iribame, Ph. (1989) «Culture et effet societal. ». Revue Frangaise de Sociologie, 32, 4 (October- December 1991), 599-614.

D'Iribarne, Ph. (2012) « Managing corporate values in diverse national cultures: the challenge of differences ». New York: Routledge. 
D'Iribarne, P. (1997) « The usefulness of an ethnographic approach to the international comparison of organization ». International Studies of Management and Organization, 26(4), 30-47.

SAADI, S Med. (1982) «Éléments d'analyse des entreprise personnelles et familiales marocaines » Revue : ISCAE, Gestion \& Sociètè $\mathrm{N}^{\circ} 9,1982$, p. 65-79.

MAMOU, R. (1985) «Motivations et comportement de l'entrepreneur marocain » Revue : ISCAE, Gestion \& Société N¹4, 1985, p. 47-66.

Soussi, Sid A. (2006) «La diversité culturelle dans les organisations : Analyse critique des fondements théoriques du management interculturel », Press : Université du Québec en Outaouais Gatineau (Qc), Canada, p. 130-143.

Nguyen, Thi Duc. (2013) «Exploring Cultural Differences in Implementing International Technology Transfer In the Case of Japanese Manufacturing Subsidiaries in Vietnam ». Contemporary Management Research, vol 9, $\mathrm{N}^{\circ} 1,03 / 2013$, p.13-34.

Dano, F. Viviane, H. Sylvie, L. (2003) «La qualité des études qualitatives: principes et pratiques » Press de l'université de droit, d'economie et des sciences d'Aix Marseille,W.P. n ${ }^{\circ} 674$, 12/2003. 